\title{
Anti-Aging Activity of Lavandula angustifolia Extract Fermented with Pediococcus pentosaceus DK1 Isolated from Diospyros kaki Fruit in UVB-Irradiated Human Skin Fibroblasts and Analysis of Principal Components
}

\author{
Ji Hoon $\mathrm{Ha}^{1}$, A Rang Kim ${ }^{1}$, Keon-Soo Lee ${ }^{1}$, Song Hua Xuan ${ }^{1}$, Hee Cheol Kang ${ }^{2}$, Dong Hwan Lee ${ }^{2}$, Mi Yeon Cha ${ }^{2}$, \\ Hye Jin $\mathrm{Kim}^{2}$, Mi An², and Soo Nam Park ${ }^{1 *}$ \\ ${ }^{1}$ Department of Fine Chemistry, Cosmetic RED Center, Cosmetic Industry Coupled Collaboration Center, Seoul National University of \\ Science and Technology, Seoul 01811, Republic of Korea \\ ${ }^{2}$ Life Science Research Institute, GFC Life Science Co. Ltd., Hwaseong 18471, Republic of Korea
}

Received: September 19, 2018/

Revised: December 24, 2018

Accepted: December 26, 2018

First published online

December 31, 2018

*Corresponding author

Phone: +82-2-970-6451;

Fax: +82-2-975-9585;

E-mail: snpark@seoultech.ac.kr

pISSN 1017-7825, eISSN 1738-8872

Copyright(C) 2019 by

The Korean Society for Microbiology and Biotechnology

\begin{abstract}
The effects of Lavandula angustifolia extract fermented with Pediococcus pentosaceus DK1 on UVB-mediated MMP-1 expression and collagen decrease in human skin fibroblasts were determined, and the conversion of its components was also analyzed. Fermentation was performed at varying L. angustifolia extract and MRS medium concentrations, and optimal fermentation conditions were selected. L. angustifolia extracts showed decreased cytotoxicity after fermentation in the fibroblasts. UVB-irradiated fibroblasts treated with fermented L. angustifolia extract showed MMP-1 expression 8.2-14.0\% lower than that in UVB-irradiated fibroblasts treated with non-fermented extract. This was observed even at fermented extract concentrations lower than those of non-fermented extracts. Fibroblasts treated with fermented L. angustifolia extract showed $20 \%$ less reduction in collagen production upon UVB irradiation than those treated with non-fermented extracts. UVB-irradiated fibroblasts treated with fermented L. angustifolia extracts showed 50\% higher inhibition of ROS generation than those treated with non-fermented extract. Luteolin and apigenin glycosides of L. angustifolia were converted during fermentation, and identified using RP-HPLC and LC/ESI-MS. Therefore, the effects of L. angustifolia extract on MMP-1 expression and collagen decrease in UVB-irradiated human skin fibroblasts were increased through fermentation by P. pentosaceus.
\end{abstract}

Keywords: Pediococcus pentosaceus DK1, fermentation, UVB, matrix metalloproteinase-1, procollagen

\section{Introduction}

The skin forms the external surface of the human body, and serves as a barrier protecting the internal organs from ultraviolet radiation, toxins, and bacteria, etc. There are two dependent layers of the skin, the epidermis and dermis, and these consist of many cells such as keratinocytes, melanocytes and fibroblasts [1]. The mechanical strength of the skin is contributed by the dermis, which is composed of the extracellular matrix (ECM) where fibroblasts synthesize ECM components such as collagen and elastin to sustain the skin's elasticity [2]. However, the structure and function of the dermis can be changed by harmful external factors such as oxidative stress, UV exposure and air pollution. These conditions accelerate aging of the skin by collapsing its dermal structure [3,4].

Ultraviolet light is a significant cause of exogenous skin damage. The ultraviolet rays that reach the Earth are classified as UVA (320-400 nm) and UVB (280-320 nm) [5]. When exposed to UV on the Earth's surface, the amount of UV radiation that reaches the human skin is known to be $25 \mathrm{~J} / \mathrm{cm}^{2}$ under natural sunlight in autumn at 
$38^{\circ} \mathrm{N}$ for $4-5 \mathrm{~h}$. It has been reported that this corresponds to ten times the minimum erythema dose in skin [6]. In particular, UVB can penetrate the upper layer of the dermis [7]. UVB exposure increases reactive oxygen species (ROS), such as hydroxyl radicals $(\bullet \mathrm{OH})$, superoxide anion radicals $\left(\mathrm{O}_{2}^{-}\right)$, singlet oxygen $\left({ }^{1} \mathrm{O}_{2}\right)$, and hydrogen peroxide $\left(\mathrm{H}_{2} \mathrm{O}_{2}\right)$. To prevent ROS-induced cellular damage, enzymes (superoxide dismutase and catalase) and non-enzymatic antioxidants (L-ascorbic acid and $\alpha$-tocopherol) are present in skin cells $[8,9]$. However, when the balance of this ROS/ antioxidant defense system is upset due to excess ROS generation caused by UVB exposure, skin cells become damaged and skin aging is accelerated $[10,11]$.

ROS induced by UVB exposure increase the expression of matrix metalloproteinase-1 (MMP-1) in fibroblasts, promoting skin photo-aging [12-14]. MMP-1 degrades collagen type 1, which is an ECM component that provides structural support to the skin. This leads to disintegration of the dermis and acceleration of skin aging [15]. Therefore, the development of anti-aging agents to inhibit UVBinduced ROS generation is an essential strategy for suppressing photo-aging [16].

Pediococcus pentosaceus as a lactobacillus is commonly found in fermented foods such as doenjang and kimchi, and is known to produce lactic acid through anaerobic fermentation [17]. Recently, it was reported that $P$. pentosaceus enhanced the immune activity of Cordyceps militaris extract, enabling it to boost the phagocytic activity of macrophages in mouse [18]. P. pentosaceus increased the anti-aging activity of Gelidium amansil extract on MMP-1 expression and decrease of collagen by UVB irradiation [19]. We isolated and identified a new lactobacillus, P. pentosaceus DK1, from Diospyros kaki fruit. P. pentosaceus DK1 donated to KCTC (KCTC12963BP) could degrade the tannins in Diospyros kaki fruit and increase the total phenol content by 1.5 fold, and the flavonoid content by 1.4 fold. Diospyros kaki fruit fermented by P. pentosaceus DK1 also enhanced the inhibitory activity of elastase for improvement of skin aging. Thus, P. pentosaceus DK1 was also used to improve the anti-aging activity of L. angustifolia.

L. angustifolia, commonly known as lavender, belongs to the Lamiaceae family [20]. Traditionally, L. angustifolia extract has been used as a remedy for neurological and rheumatic diseases, due to its antibacterial and relaxing properties, and is mainly obtained by extracting volatile components from oils [21, 22]. Another method involves extracting L. angustifolia using water or ethanol, which has been reported to produce extracts that show antioxidant properties, whitening effect, and inhibitory effect on sebum production [23]. The major components of L. angustifolia extract are linalool, linalyl acetate, ladanein, apigenin, apigenin-7-O- $\beta$-glucoside, luteolin, luteolin-7-O- $\beta$-glucoside, and 5, $4^{\prime}$-dihydroxy flavonoid-7-O- $\beta$-pyranglycuronate butyl ester [24, 25]. Recently, Ahn, et al. [26] reported that the antioxidant, tyrosinase, and elastase inhibitory activities of L. angustifolia extract, as well as the ratios of phenolic compounds such as rosmarinic acid, were significantly increased by natural fermentation. However, their fermentation method was very old, and the microorganisms used were also not identified. There have been no studies so far on the anti-aging activity and composition analysis of L. angustifolia extracts fermented by P. pentosaceus DK1.

In this study, the anti-aging effects of L. angustifolia extract fermented by $P$. pentosaceus DK1 were evaluated on MMP-1 expression, collagen production, and antioxidant activity, and analyzed on the converted components during fermentation.

\section{Materials and Methods}

\section{Reagents and Chemicals}

Human skin fibroblasts (HS68 cells) were purchased from Lonza (Basel, Switzerland). Dulbecco's modified Eagle's medium (DMEM), foetal bovine serum (FBS), trypsin, and penicillin-streptomycin were obtained from Capricorn Scientific (Ebsdorfergrund, Germany).

\section{Preparation of L. angustifolia Extract}

Fermented and non-fermented L. angustifolia extract were obtained from GFC Life Science (Korea). The dried L. angustifolia flowers $(2 \mathrm{~kg}$ ) were homogenized in $70 \%$ ethanol (40 L) for 20 days at room temperature. The extract powder $(410.93 \mathrm{~g})$ was obtained by filtration and vacuum evaporation drying

\section{Fermentation of L. angustifolia Extract}

In order to cultivate the P. pentosaceus DK1 strain, lactobacilli MRS broth (BD 288130) purchased from Difco (USA) was selected as the culture medium for the optimal growth conditions of the strain. MRS medium was developed to favor the growth of lactobacilli in 1960 by De Man, Rogosa and Sharpe and is known to support the growth of lactobacilli including Pediococcus. MRS broth was dissolved in distilled water, and L. angustifolia extract was added (Table 1). P. pentosaceus DK1 was pre-cultured in MRS broth and cultured at $37^{\circ} \mathrm{C}$ for $24 \mathrm{~h}$. Then, $10 \%$ of the strains precultured in the broth containing the L. angustifolia extract were cultured at $37^{\circ} \mathrm{C}$ for $14 \mathrm{~d}$. The fermented broth was then centrifuged at 9,010 $\mathrm{g}$ for $15 \mathrm{~min}$ and the supernatant was filtered using a $0.22 \mu \mathrm{m}$ cellulose filter. Finally, the fermented broth was fractioned with ethyl acetate. The ethyl acetate fraction was evaporated to obtain fermented L. angustifolia extract. All the processes were indicated in Scheme 1. 
Table 1. Fermentation conditions of L. angustifolia extract.

\begin{tabular}{ccc}
\hline \multirow{2}{*}{$\begin{array}{c}\text { Sample } \\
\text { Group }\end{array}$} & \multicolumn{2}{c}{ Fermentation condition } \\
\cline { 2 - 3 } & $\begin{array}{c}\text { L. angustifolia extract } \\
\text { Concentration (\%) }\end{array}$ & $\begin{array}{c}\text { MRS medium } \\
\text { Ratio (\%) }\end{array}$ \\
\hline A1 & $0.1 \%$ & $0.69 \%$ \\
A2 & $0.1 \%$ & $1.38 \%$ \\
A3 & $0.1 \%$ & $2.75 \%$ \\
A4 & $0.1 \%$ & $5.50 \%$ \\
B1 & $0.5 \%$ & $0.69 \%$ \\
B2 & $0.5 \%$ & $1.38 \%$ \\
B3 & $0.5 \%$ & $2.75 \%$ \\
B4 & $0.5 \%$ & $5.50 \%$ \\
\hline
\end{tabular}

\section{Cell Culture}

Human skin fibroblasts (HS68 cells) were incubated in DMEM medium supplemented with $10 \% \mathrm{FBS}, 100 \mathrm{U} / \mathrm{ml}$, of penicillin and $100 \mu \mathrm{g} / \mathrm{ml}$ of streptomycin. The cells were incubated in medium at $37^{\circ} \mathrm{C}$ in a humid incubator under $5 \% \mathrm{CO}_{2}$ atmosphere.

\section{UVB Radiation}

For supply of UVB radiation, a CL-1000 Ultraviolet Crosslinker (UVP, USA), with an emission spectrum of $280-370 \mathrm{~nm}$ and a peak at $312 \mathrm{~nm}$, was used. HS68 cells with $70-80 \%$ confluence were treated with fermented and non-fermented L. angustifolia extract at various concentrations in a plastic, $60-\mathrm{mm}$ Petri dish in FBS-free medium for $24 \mathrm{~h}$. The medium was then removed, and the cells were rinsed twice with phosphate-buffered saline (PBS). The cells were irradiated through the cover of the dish by UVB after addition of $1 \mathrm{~mL}$ PBS in the wells (height; $3 \mathrm{~mm}$ ). After UV exposure, the medium was replaced with FBS-free medium.

\section{Cell Viability}

A 3-(4,5-dimethyl-2-thiazolyl)-2,5-diphenyltetrazolium bromide (MTT, Sigma-Aldrich, USA) assay was used to analyze cell viability. HS68 cells were seeded in 96-well plates at $1 \times 10^{4}$ cells per well and incubated for $24 \mathrm{~h}$ at $37^{\circ} \mathrm{C}$. After treatment with various doses of fermented or non-fermented L. angustifolia extract for $24 \mathrm{~h}$ or exposure to UVB radiation, the medium was replaced with FBS-free medium and the cells were further incubated for up to $72 \mathrm{~h}$. Subsequently, $0.5 \mathrm{mg} / \mathrm{ml}$ MTT solution was added and the cells were further incubated for $30 \mathrm{~min}$ at $37^{\circ} \mathrm{C}$. Then, $100 \mu$ dimethyl sulfoxide (DMSO) was added to the wells respectively, and the absorbance was determined at $570 \mathrm{~nm}$ using a microplate reader (TECAN, Austria).

\section{Quantitative Analysis of MMP-1 Expression}

HS-68 fibroblasts were incubated up to $70-80 \%$ confluence on $60-\mathrm{mm}$ plates in a humid incubator maintained at $37^{\circ} \mathrm{C}$. After treatment with different concentrations of the fermented or nonfermented L. angustifolia extract, the cells were exposed to $80 \mathrm{~mJ} / \mathrm{cm}^{2}$ UVB under PBS and then incubated further for $48 \mathrm{~h}$ in fresh medium without FBS. Secreted MMP-1 proteins within the culture medium were measured by an ELISA kit (R\&D Systems, USA) according to the manufacturer's instruction.

\section{HPLC and LC/ESI-MS Analysis}

HPLC analysis of the lavender was carried out using a Shimadzu LC-20A HPLC system (Shimadzu, Japan) equipped with a UVD 170s DIONEX detector and Shim-pack VP-ODS C18 column (L: $250 \mathrm{~mm}$, LD: $4.6 \mathrm{~mm}, 5 \mu \mathrm{m})$. The mobile phase was composed of A ( $2 \%$ acetic acid in $\left.\mathrm{H}_{2} \mathrm{O}\right)$ and $\mathrm{B}(0.5 \%$ acetic acid in $50 \%$ acetonitrile aqueous solution). The working conditions were as follows: 0-25 $\min , 0 \%(\mathrm{v} / \mathrm{v})$ of $\mathrm{B} ; 25-40 \mathrm{~min}, 0-25 \%(\mathrm{v} / \mathrm{v})$ of $\mathrm{B} ; 40-80 \mathrm{~min}, 25-$ $35 \%(\mathrm{v} / \mathrm{v})$ of $\mathrm{B} ; 80-110 \mathrm{~min}, 35-40 \%(\mathrm{v} / \mathrm{v})$ of $\mathrm{B} ; 110-120 \mathrm{~min}, 40-$ $20 \%(\mathrm{v} / \mathrm{v})$ of $\mathrm{B} ; 120-130 \mathrm{~min}, 20-10 \%$ (v/v) of $\mathrm{B} ; 130-140 \mathrm{~min}, 10-$ $0 \%(\mathrm{v} / \mathrm{v})$ of $\mathrm{B} ; 140-150 \mathrm{~min}, 0 \%(\mathrm{v} / \mathrm{v})$ of $\mathrm{B}$. The flow rate was $1.0 \mathrm{ml} / \mathrm{min}$ and the samples were observed at $365 \mathrm{~nm}$. The samples were passed through a 0.2 -um filter and then $20 \mu \mathrm{l}$ of the samples with $10,000 \mu \mathrm{g} / \mathrm{ml}$ were injected into the HPLC.

The mass spectrometric analysis was performed using an LCQ Ion Trap Mass Spectrometer (Thermo Finnigan, USA) with an ESI interface and detection was done in positive ion mode by the National Instrumentation Center for Environmental Management College of Seoul National University (Seoul, Korea). The operating

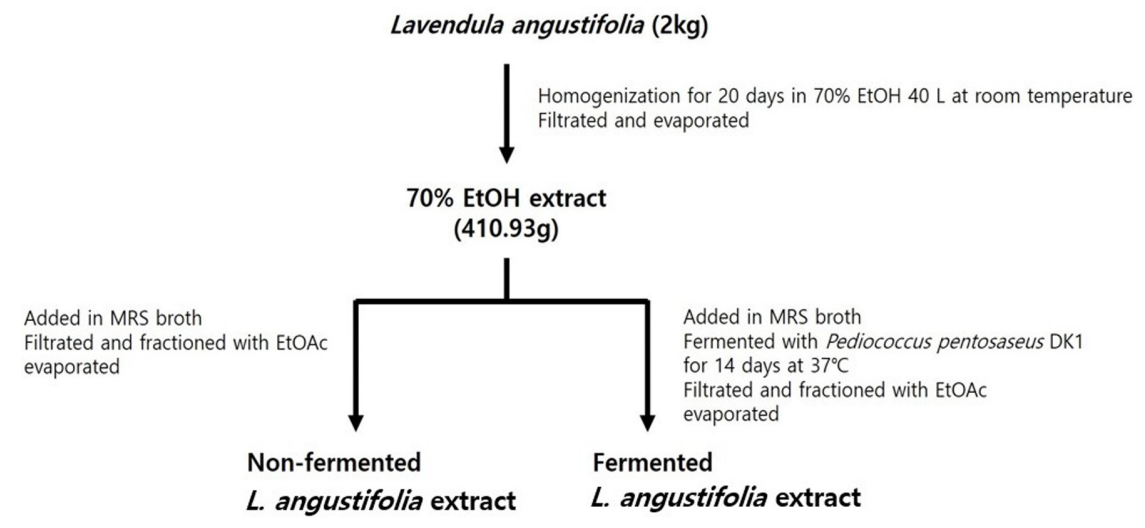

Scheme 1. Preparation of fermented and non-fermented L. angustifolia extracts. 

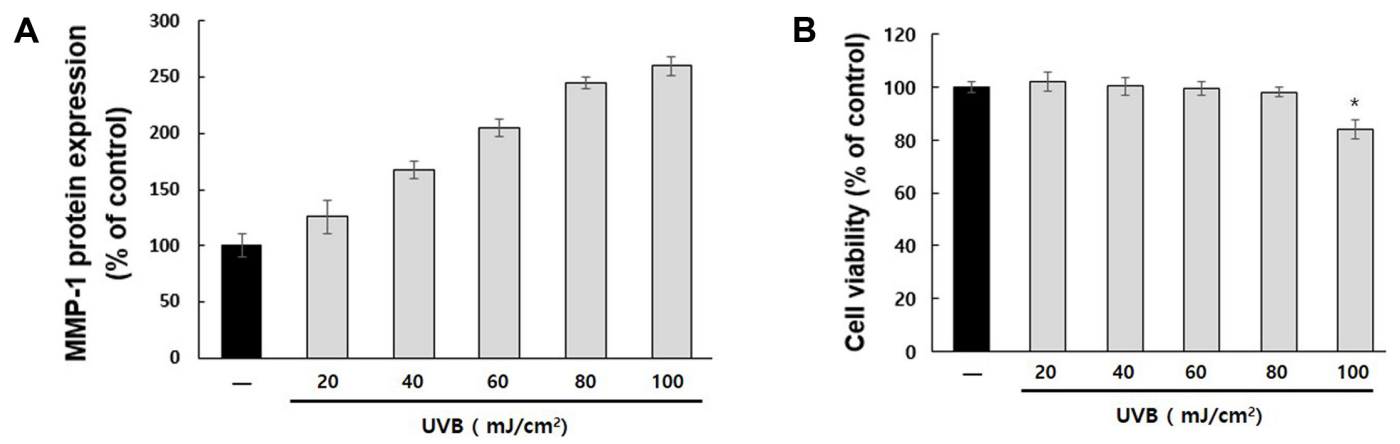

Fig. 1. Effects of UVB irradiation on human skin fibroblasts. The cells were irradiated with various doses of UVB and incubated $72 \mathrm{~h}$. Then, the levels of MMP-1 proteins (A) and cell viability (B) were measured. Data were presented as mean \pm SD of three independent experiments. ${ }^{*} p<0.01$ compared with the negative control.

conditions were as follows: capillary voltage, $33 \mathrm{~V}$; capillary temperature, $400^{\circ} \mathrm{C}$; nebuliser pressure, $10 \mathrm{psi}$; and drying gas, $\mathrm{N}_{2}$. Compounds within STE were identified by comparing the UV spectra, retention times, and fragment ions of standard materials.

\section{Intracellular ROS Evaluation}

The intracellular ROS levels were evaluated using the fluorescence dye $2^{\prime}, 7^{\prime}$-dichlorodihydrofluorescein diacetate $\left(\mathrm{H}_{2} \mathrm{DCF}-\mathrm{DA}\right.$, SigmaAldrich, USA), which is generated by the conversion of nonfluorescent $\mathrm{H}_{2} \mathrm{DCF}-\mathrm{DA}$ to highly fluorescent 2 ', $7^{\prime}$-dichlorofluorescein (DCF) by intracellular ROS. HS-68 cells were incubated with $20 \mu \mathrm{M} \mathrm{H} \mathrm{H}_{2}$ DCFH-DA for $30 \mathrm{~min}$ at $37^{\circ} \mathrm{C}$. The cells were then washed twice with PBS and irradiated by $80 \mathrm{~mJ} / \mathrm{cm}^{2}$ UVB. Fluorescence signals were detected using a fluorescence ELISA reader (Perkin Elmer, USA; excitation, $490 \mathrm{~nm}$; emission, $530 \mathrm{~nm}$ ).

\section{Statistical Analysis}

All statistical analyses were performed using SPSS 17.0 (SPSS, USA) software. The results were presented as mean \pm standard deviation (SD). Statistically significant differences were calculated by one-way ANOVA, where $p<0.01$ was considered statistically significant.

\section{Results}

Effect of Fermented L. angustifolia Extract on UVBIncreased MMP-1 Expression in Human Skin Fibroblasts

UVB-treated fibroblasts secrete a greater amount of matrix metalloproteinase-1 (MMP-1) than untreated fibroblasts. MMP-1 is a metalloproteinase with a zinc ion in its center, and promotes skin aging through degradation of collagen type I and III, which act as support fixtures in the dermis.

We studied the effects of fermented and non-fermented L. angustifolia extracts on UVB-induced MMP-1 expression in human fibroblasts. To design an optimized system for measurement of anti-aging activity, the fibroblasts were irradiated by UVB at $20-100 \mathrm{~mJ} / \mathrm{cm}^{2}$. As the UVB irradiation dose was increased, the amount of MMP-1 expression in the UVB-exposed fibroblasts also increased (Fig. 1A). However, at $100 \mathrm{~mJ} / \mathrm{cm}^{2}$, there was a slight decrease in cell viability (Fig. 1B). We then set the ultraviolet radiation (UVB) at $80 \mathrm{~mJ} / \mathrm{cm}^{2}$, which produced a high MMP-1 expression level without cytotoxicity in the fibroblasts.

Fermented and non-fermented L. angustifolia extracts (A1-B4) were prepared under various medium conditions, using the concentrations (Table 1) of L. angustifolia extract $(0.1 \%$ or $0.5 \%)$ and the concentrations of MRS medium $(0.69 \%, 1.38 \%, 2.75 \%$, or $5.50 \%)$ as described in Materials and Methods.

In order to select the optimal fermentation conditions for the L. angustifolia extract in terms of anti-aging activity, $10 \mu \mathrm{g} / \mathrm{ml}$ of L. angustifolia extract fermented product and non-fermented product were used to treat fibroblasts for $24 \mathrm{~h}$ and these were then irradiated with $80 \mathrm{~mJ} / \mathrm{cm}^{2} \mathrm{UVB}$. As shown in Fig. 2A, all fermented and non-fermented L. angustifolia extracts reduced the increased MMP-1 protein expression under UVB irradiation. Fermented L. angustifolia extract showed a greater inhibitory effect on MMP-1 expression than non-fermented L. angustifolia extract. In particular, the inhibitory effect of MMP-1 expression of the L. angustifolia extract fermented at B2 condition $(0.5 \%$ of L. angustifolia extract and $1.38 \%$ of MRS medium) was the largest of all extracts. All extracts fermented at all conditions showed no cytotoxicity (data not shown). We selected this condition as optimal for fermentation and used these same conditions in subsequent experiments.

As shown in Fig. 2B, non-fermented L. angustifolia extracts indicated cytotoxicity at more than $50 \mu \mathrm{g} / \mathrm{ml}$ but 
A

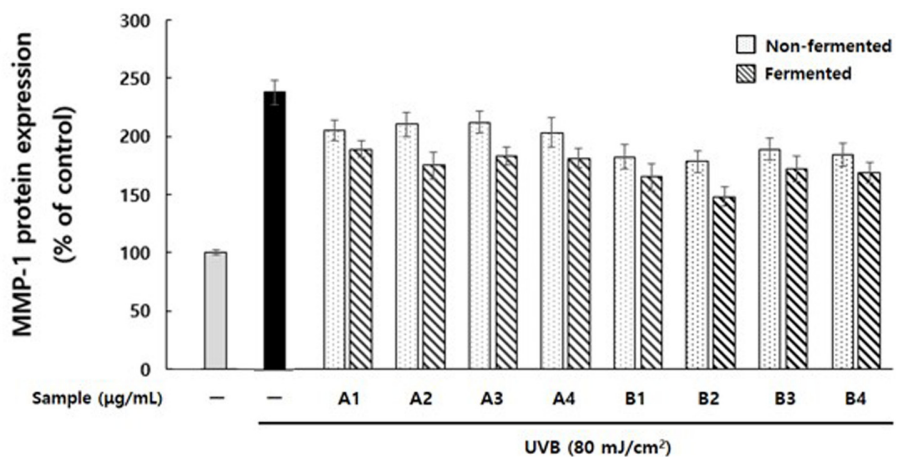

$\mathbf{B}$

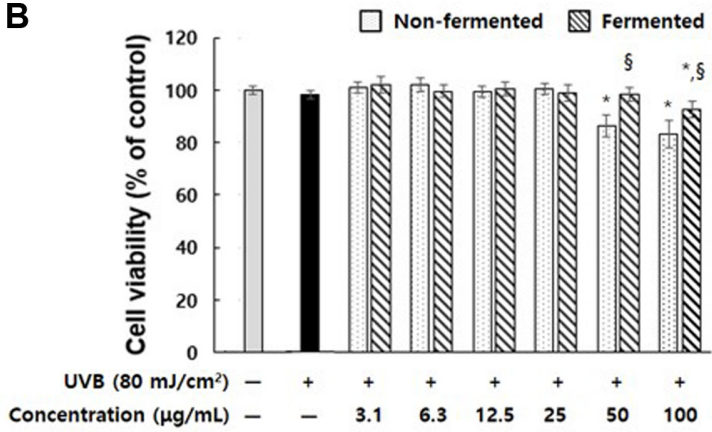

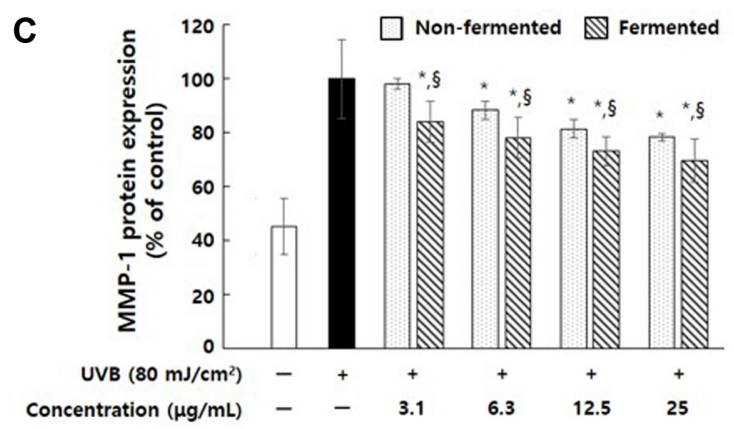

Fig. 2. Effect of fermented and non-fermented L. angustifolia extracts on UVB-induced MMP-1 expression in human skin fibroblasts. (A) MMP-1 expression was determined after pre-treatment with $10 \mu \mathrm{g} / \mathrm{ml}$ of fermented and non-fermented L. angustifolia extracts and irradiation of $80 \mathrm{~mJ} / \mathrm{cm}^{2}$ UVB. The cell viability (B) and MMP-1 expression (C) were determined after pre-treatment of fermented and non-fermented L. angustifolia extracts at indicated concentration and irradiation of $80 \mathrm{~mJ} / \mathrm{cm}^{2}$ UVB. Data are presented as the mean \pm SD of three independent experiments. ${ }^{*} p<0.01$ compared with the UVB-treated control. ${ }^{\circledR} p<0.01$ compared with cells treated with non-fermented L. angustifolia extract.

fermented L. angustifolia extracts decreased the cytotoxicity at the same concentrations. In particular, fermented $L$. angustifolia extract showed no cytotoxicity up to $50 \mu \mathrm{g} / \mathrm{ml}$. We selected $25 \mu \mathrm{g} / \mathrm{ml}$ as the maximal concentration for further experiments.

Fermented and non-fermented L. angustifolia extracts decreased UVB irradiation-increased MMP-1 expression in a dose-dependent manner (Fig. 2C). The MMP-1 expression levels of fermented L. angustifolia extracts were 8.2-14.0\% lower than those of non-fermented L. angustifolia extracts at all concentrations $(3.1-25 \mu \mathrm{g} / \mathrm{ml})$. These results suggest that L. angustifolia extracts fermented by P. pentosaceus DK1 are effective in the suppression of MMP-1 protein expression increased by UVB irradiation.

\section{Effect of Fermented L. angustifolia Extract on Collagen Production}

When the skin is exposed to UVB, the levels of type 1 procollagen decrease. Anti-aging agents can protect against the degradation of collagen in UVB-exposed fibroblasts. The effects of fermented and non-fermented L. angustifolia extract were evaluated on UVB irradiation-induced decrease in collagen production (Fig. 3).

In UVB-irradiated fibroblasts, the level of procollagen type 1 was $42 \%$ lower than the levels of collagen in normal cells without UVB irradiation. Fermented and nonfermented L. angustifolia extracts increased the levels of collagen reduced by UVB irradiation in a dose-dependent manner. Fermented L. angustifolia extracts presented a $20 \%$ higher level of collagen production than non-fermented L. angustifolia extracts at 12.5 and $25 \mu \mathrm{g} / \mathrm{ml}$. However, treatment with fermented and non-fermented L. angustifolia extracts without UVB irradiation showed no increase in collagen production in fibroblasts (data not shown). These results suggest that the fermentation of L. angustifolia extracts recover collagen production after reduction from UVB irradiation.

\section{Effect of Fermented L. angustifolia Extract on UVB- Induced ROS Generation}

UVB irradiation increases ROS generation in cells and ROS-stimulated fibroblasts increases expression of MMP-1 protein and inhibits collagen production [27]. Therefore, it is important to identify the active compounds that are able 


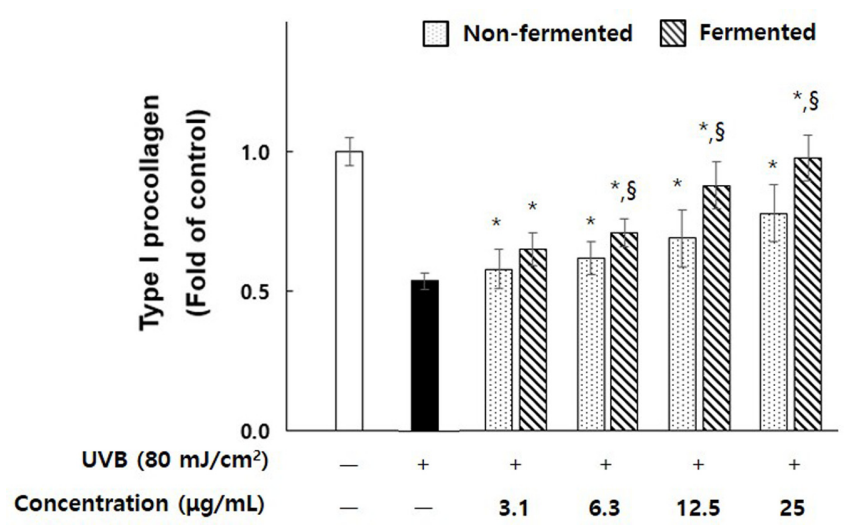

Fig. 3. Effect of fermented and non-fermented L. angustifolia extracts on UVB-mediated production of procollagen type I in human skin fibroblasts.

Data are presented as the mean \pm SD of three independent experiments. ${ }^{*} p<0.01$ compared with the UVB-treated control. ${ }^{\circledR} p<0.01$ compared with cells treated with non-fermented L. angustifolia extract.

to inhibit ROS generation stimulated by UVB for anti-aging activity [28]. $\mathrm{H}_{2}$ DCF-DA [29] was used to determine the effect of fermented and non-fermented L. angustifolia extracts on UVB-induced ROS generationin fibroblasts.

As shown in Fig. 4A, fibroblasts irradiated with UVB showed a $421.2 \% \pm 20.4 \%$ higher ROS generation than cells without UVB irradiation. At all concentrations (3.1-25 $\mu \mathrm{g} / \mathrm{ml})$, fermented L. angustifolia extracts reduced the amount of ROS generation by more than $16 \%$ compared to those of non-fermented L. angustifolia extracts. Non-fermented and fermented L. angustifolia extracts at $6.3 \mu \mathrm{g} / \mathrm{ml}$ indicated $335.4 \% \pm 11.3 \%$ and $283.2 \% \pm 14.8 \%$ of ROS levels on UVBirradiated fibroblasts.

ROS levels of fermented L. angustifolia extract at $25 \mu \mathrm{g} / \mathrm{ml}$ were $116.3 \% \pm 12.5 \%$ lower than that in the non-fermented L. angustifolia extract (70.6\%) (Figs. 4A and 4B). These results suggest that fermentation using $P$. pentosaceus DK1 enhances the inhibitory effect of L. angustifolia extract on UVB-induced ROS generation.

\section{Component Analysis of L. angustifolia Extract before and after Fermentation}

Changes in the composition of natural products fermented by microorganisms can affect their anti-aging activity [31]. In this study, the anti-aging activity of L. angustifolia extract was increased after fermentation, with reduced ROS generation, decreased MMP-1 expression, and increased collagen production.

We observed the changes in the components of the L. angustifolia extract after fermentation by $P$. pentosaceus DK1. The components were identified by HPLC, UVspectrophotometry, and LC/ESI-MS (Table 2), and quantified using standard materials to confirm the change in the amounts of components. As shown in Fig. 5, luteolin-7-O-
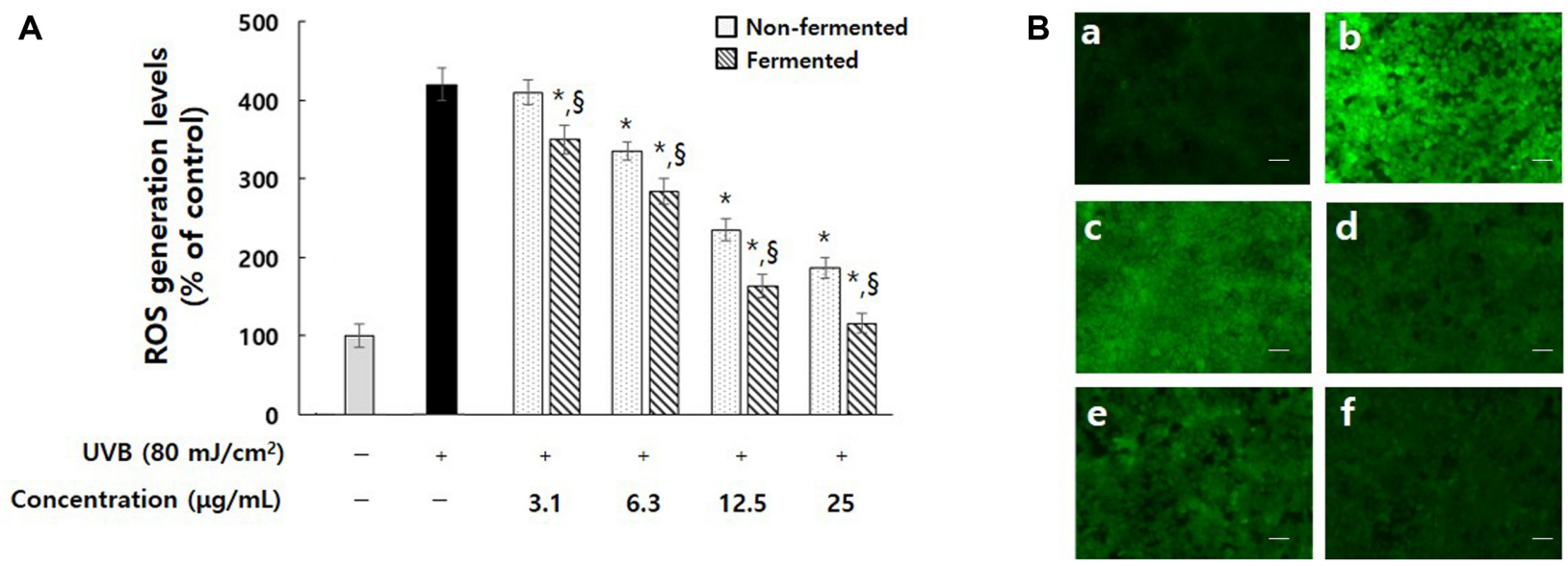

Fig. 4. Effect of fermented and non-fermented L. angustifolia extracts on UVB-induced ROS generation in human skin fibroblasts. The cells were treated with different concentrations of fermented and non-fermented L. angustifolia extracts for $24 \mathrm{~h}$ and then treated with $20 \mu \mathrm{M}$ $\mathrm{H}_{2} \mathrm{DCFH}$-DA for $30 \mathrm{~min}$. Subsequently, the cells were irradiated with $80 \mathrm{~mJ} / \mathrm{cm}^{2} \mathrm{UVB}$. ROS generation was measured using a fluorescence reader (A) and fluorescence microscope (B). In Fig. 4B, the cells were treated with (a) non-treatment; (b) $80 \mathrm{~mJ} / \mathrm{cm}^{2} \mathrm{UVB}$ irradiation; treatment of 6.3 (c) and $25 \mu \mathrm{g} / \mathrm{ml}$ (d) non-fermented L. angustifolia extract and UVB irradiation; treatment of 6.3 (e) and $25 \mu \mathrm{g} / \mathrm{ml}$ (f) fermented L. angustifolia extract and UVB irradiation. Scale bar, $50 \mu \mathrm{m}$. Data are presented as the mean $\pm \mathrm{SD}$ of three independent experiments. ${ }^{*} p<0.01$ compared with the UVBtreated control. ${ }^{s_{p}}<0.01$ compared with cells treated with non-fermented L. angustifolia extract. 
Table 2. Mass and UV spectrum of identified compounds in L. angustifolia extract fermented or non-fermented by P. pentosaceus DK1.

\begin{tabular}{|c|c|c|c|c|c|c|}
\hline \multirow{2}{*}{$\begin{array}{c}\text { HPLC } \\
\text { Peak No. }\end{array}$} & \multirow[b]{2}{*}{ Name of the compound } & \multirow{2}{*}{$\begin{array}{l}\text { Molecular } \\
\text { formula }\end{array}$} & \multirow{2}{*}{$\begin{array}{l}\text { Retention time } \\
\qquad(\min )\end{array}$} & \multicolumn{3}{|c|}{ Measurement } \\
\hline & & & & $\lambda_{\max }(\mathrm{nm})$ & $\begin{array}{l}\text { Negative ions }(m / z) \\
{[\mathrm{M}+\mathrm{H}]^{-}}\end{array}$ & $\begin{array}{l}\text { Positive ions }(\mathrm{m} / \mathrm{z}) \\
{[\mathrm{M}+\mathrm{H}]^{+}}\end{array}$ \\
\hline 1 & Chlorogenic acid & $\mathrm{C}_{16} \mathrm{H}_{18} \mathrm{O}_{9}$ & 49.754 & 244,322 & 353.4 & 355.1 \\
\hline 2 & Luteoln-7-O-glucuronide & $\mathrm{C}_{21} \mathrm{H}_{18} \mathrm{O}_{12}$ & 69.408 & 253,347 & - & 463.8 \\
\hline 3 & Luteolin-7-O-glucoside & $\mathrm{C}_{21} \mathrm{H}_{20} \mathrm{O}_{11}$ & 80.261 & 254,346 & 447.9 & 449.4 \\
\hline 4 & Rosmarinic acid & $\mathrm{C}_{18} \mathrm{H}_{15} \mathrm{O}_{8}$ & 82.956 & 290,331 & 359.2 & - \\
\hline 5 & Apigenin-7-O-glucoside & $\mathrm{C}_{21} \mathrm{H}_{20} \mathrm{O}_{10}$ & 92.844 & 267,334 & 431.8 & 433.8 \\
\hline 6 & Luteolin & $\mathrm{C}_{15} \mathrm{H}_{10} \mathrm{O}_{6}$ & 98.003 & 254.349 & 285.2 & 287.4 \\
\hline 7 & Apigenin & $\mathrm{C}_{15} \mathrm{H}_{10} \mathrm{O}_{5}$ & 106.566 & 270,334 & 269.1 & 271.2 \\
\hline
\end{tabular}

glucoside (peak 3) and apigenin-7-O-glucoside (peak 5) in fermented L. angustifolia extract were decreased compared with the non-fermented L. angustifolia extract and luteolin (peak 6) and apigenin (peak 7), as their aglycone components, were increased, respectively. In addition, chlorogenic acid (peak 1) decreased after fermentation. Interestingly, there was no significant change in luteolin 7-O-glucuronide (peak 2) after fermentation, despite having a similar structure to luteolin-7-O-glucoside.

A

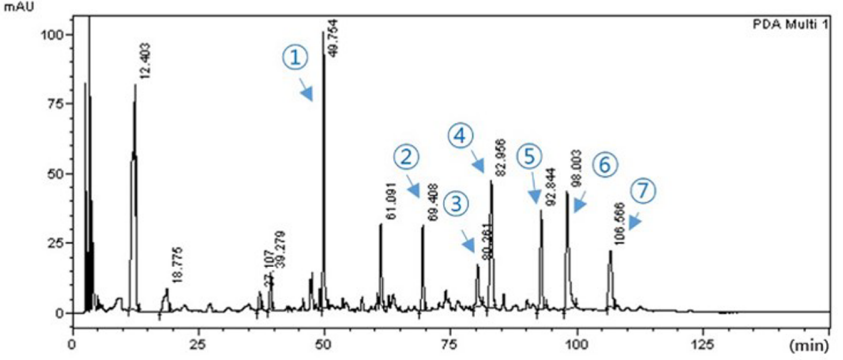

B

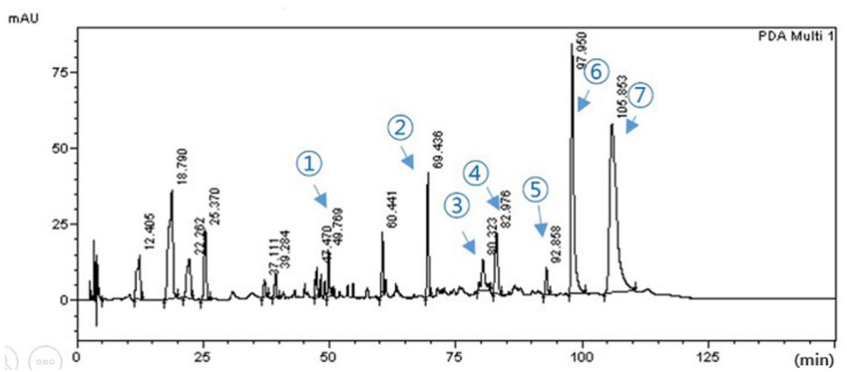

Fig. 5. HPLC chromatograms of $L$. angustifolia extracts fermented (A) and non-fermented (B).

The peaks were detected at $365 \mathrm{~nm}$ of wavelength for $150 \mathrm{~min}$.

\section{Anti-Aging Effect of the Identified Compounds in UVB- Irradiated Human Skin Fibroblasts}

Identified components of the L. angustifolia extract converted by P. pentosaceus DK1 were evaluated on UVBinduced ROS generation, MMP-1 expression and collagen degradation. UVB was irradiated to human fibroblasts after treatment with luteolin, luteolin-7-O-gluconisde, apigenin and apigenin-7-O-glucoside.

Luteolin and apigenin reduced $80 \%$ and $85.8 \%$ of ROS generation increased by UVB irradiation compared to their glycosides (Fig. 6A). Luteolin and apigenin also reduced $26.5 \%$ and $22.4 \%$ of UVB-enhanced MMP-1 levels less than their glycosides, respectively (Fig. 6B). In UVB-reduced collagen production, luteolin and apigenin increased 9.5\% and $12.5 \%$ more than their glycosides (Fig. 6C).

Taken together, these results suggest that aglyconation of the flavonoids in L. angustifolia extract through fermentation by $P$. pentosaceus DK1 is a major factor in promoting antiaging activity through a reduction in UVB-induced ROS production.

\section{Discussion}

Recently, fermentation using microorganisms and enzymes has been shown to enhance both the safety and activity of natural products for cosmetics agents [31]. The mechanism of efficacy enhancement of materials by microbial fermentation is as follows: 1) the microorganism breaks down the substance to a small size to increase its absorption into the skin; 2) the microorganism enhances the product's physiological activities by removing glycoside; 3) favorable nutrients including vitamins and organic acids are produced during microbial cultivation [32, 33]. In addition, heavy metals and pesticide residues in the extracts of raw plants 
A

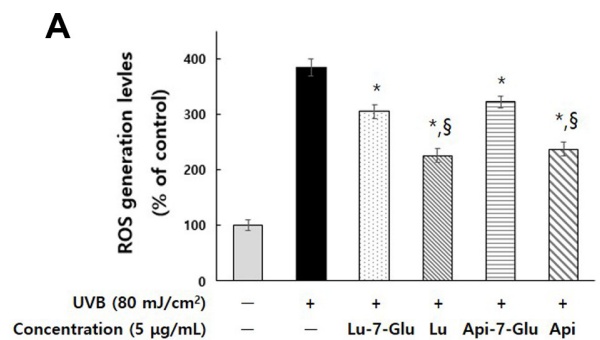

B

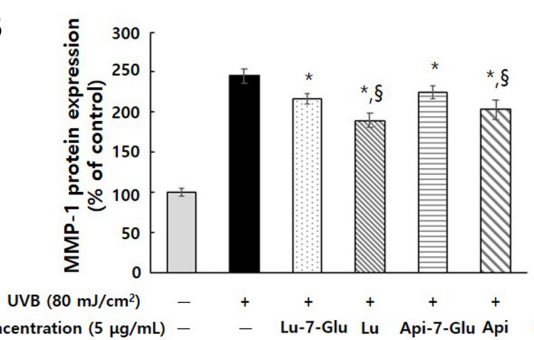

C

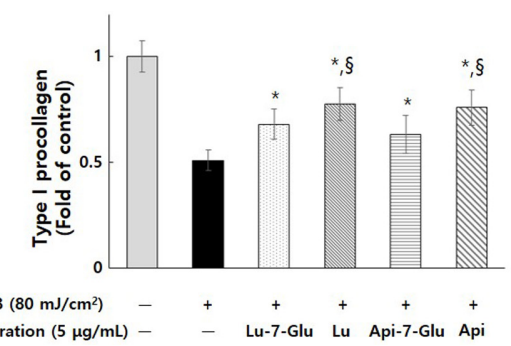

Fig. 6. Effect of identified compounds on ROS generation, MMP-1 expression and collagen production in UVB-irradiated human skin fibroblasts.

Data are presented as mean $\pm \mathrm{SD}$ of three independent experiments. ${ }^{*} p<0.01$ compared with the negative control. ${ }^{\S} p<0.01$ compared with cells treated with non-fermented L. angustifolia extract.

are harmful to the skin, microorganisms can enhance the safety of the extracts through decomposition and/or adsorption [34].

In this study, L. angustifolia extract was fermented using P. pentosaceus DK1, a new microorganism isolated from Diospyros kaki fruit, commonly known as persimmon, to develop a novel material for the inhibition of skin-aging caused by UVB irradiation. We optimized the fermentation conditions using P. pentosaceus DK1 in $1.38 \%$ MRS medium supplemented with $0.5 \%$ L. angustifolia extract. Fermentation of L. angustifolia extract by P. pentosaceus DK1 increased the anti-aging activities while decreasing MMP-1 expression and ROS generation, and increased collagen production in UVB-irradiated human skin fibroblasts. We identified that the flavonoid glycosides, luteolin-7-O-glucoside and apigenin-7-O-glucoside, contained in L. angustifolia extract were decreased and the contents of sugar-removed luteolin and apigenin after fermentation were increased. These results suggest that $P$. pentosaceus DK1 may remove glucosides from flavonoid glucosides as well as degradation of tannins. Luteolin and apigenin indicated higher antiaging activities than the glucosides of them on MMP-1 expression, collagen production and ROS generation in UVB-irradiated fibroblasts. Sung, et al. reported that luteolin had a higher antioxidant activity than the glycoside in intracellular systems [35]. Luteolin and apigenin were also reported to suppress UV-induced MMP-1 expression through anti-oxidative activity on human skin fibroblasts [36] and human keratinocytes (HaCaT cells) [37]. Apigenin increases collagen synthesis in fibroblasts [38].

In conclusion, $P$. pentosaceus DK1 could increase the antioxidative and aging activities of the L. angustifolia extract through bioconversion which increases the levels of apigenin and luteolin by aglyconation of their glucosides. Thus, L. angustifolia extract fermented by $P$. pentosaceus DK1 could have applicability as an anti-aging agent.

\section{Acknowledgments}

This study was supported by a grant of the Korean Health Technology R\&D Project, Ministry of Health \& Welfare, Republic of Korea (Grant No. HN15C0104).

\section{Conflict of Interest}

The authors have no financial conflicts of interest to declare.

\section{References}

1. MacNeil S. 2007. Progress and opportunities for tissueengineered skin. Nature 445: 874-880.

2. Hussain SH, Limthongkul B, Humphreys TR. 2013. The biomechanical properties of the skin. Dermatol. Surg. 39: 193203.

3. D'Orazio J, Jarrett S, Amaro-Ortiz A, Scott T. 2013. UV radiation and the skin. Int. J. Mol. Sci. 14: 12222-12248.

4. Rittie L, Fisher GJ. 2002. UV-light-induced signal cascades and skin aging. Ageing Res. Rev. 1: 705-720.

5. Ramachandran S, Prasad NR. 2008. Effect of ursolic acid, a triterpenoid antioxidant, on ultraviolet-B radiation-induced cytotoxicity, lipid peroxidation and DNA damage in human lymphocytes. Chem. Biol. Interact. 176: 99-107.

6. Packer L. 1994. Ultraviolet radiation(UVA,UVB) and skin antioxidants. New Compr. Biochem. 28: 239-255.

7. Maeda K. 2018. Analysis of ultraviolet radiationwavelengths causing hardening and reduced elasticity of collagen gels in vitro. Cosmetics 5: 1-14.

8. Birben E, Sahiner UM, Sackesen C, Erzurum S, Kalayci O. 2012. Oxidative stress and antioxidant defense. World Allergy Organ. J. 5: 9-19.

9. Stanczyk M, Gromadzinska J, Wasowicz W. 2005. Roles of reactive oxygen species and selected antioxidants in regulation of cellular metabolism. Int. J. Occup. Med. Environ. Health 18: 15-26.

10. Nita M, Grzybowski A. 2016. The role of the reactive 
oxygen species and oxidative stress in the pathomechanism of the age-related ocular diseases and other pathologies of the anterior and posterior eye segments in adults. Oxid. Med. Cell Longev. 2016: 3164734.

11. Poljsak B, Suput D, Milisav I. 2013. Achieving the balance between ROS and antioxidants: when to use the synthetic antioxidants. Oxid. Med. Cell Longev. 2013: 956792.

12. Khan MN, Mobin M, Abbas ZK, AlMutairi KA, Siddiqui ZH. 2017. Role of nanomaterials in plants under challenging environments. Plant Physiol. Biochem. 110: 194-209.

13. Chekulayeva LV, Shevchuk IN, Chekulayev VA, Ilmarinen K. 2006. Hydrogen peroxide, superoxide, and hydroxyl radicals are involved in the phototoxic action of hematoporphyrin derivative against tumor cells. J. Environ. Pathol. Toxicol. Oncol. 25: 51-77.

14. Moon HJ, Lee SR, Shim SN, Jeong SH, Stonik VA, Rasskazov VA, et al. 2008. Fucoidan inhibits UVB-induced MMP-1 expression in human skin fibroblasts. Biol. Pharm. Bull. 31: 284-289.

15. Fisher GJ, Quan T, Purohit T, Shao Y, Cho MK, He T, et al. 2009. Collagen fragmentation promotes oxidative stress and elevates matrix metalloproteinase- 1 in fibroblasts in aged human skin. Am. J. Pathol. 174: 101-114.

16. Binic I, Lazarevic V, Ljubenovic M, Mojsa J, Sokolovic D. 2013. Skin ageing: natural weapons and strategies. Evid. Based Complement Alternat. Med. 2013: 827248.

17. Jonganurakkun B, Wang Q, Xu SH, Tada Y, Minamida K, Yasokawa D, et al. 2008. Pediococcus pentosaceus NB-17 for probiotic use. J. Biosci. Bioeng. 106: 69-73.

18. Kwon HK, Jo WR, Park HJ. 2018. Immune-enhancing activity of C. militaris fermented with Pediococcus pentosaceus (GRC-ON89A) in CY-induced immunosuppressed model. BMC Complement. Altern. Med. 18: 75.

19. Park SD, Lee DE, Jeong JW, Kim YT, Kim HM, Kim YJ, et al. 2014. Comprising lactic acid fermentation product of Gelidium amansil extract as an active ingredient for improving skin wrinkle. J. Microbiol Biotechnol. 24: 1583-1591.

20. Woronuk G, Demissie Z, Rheault M, Mahmoud S. 2011. Biosynthesis and therapeutic properties of Lavandula essential oil constituents. Planta Med. 77: 7-15.

21. Lopez V, Nielsen B, Solas M, Ramirez MJ, Jager AK. 2017. Exploring pharmacological mechanisms of lavender (Lavandula angustifolia) essential oil on central nervous system targets. Front Pharmacol. 8: 280.

22. Cavanagh HM, Wilkinson JM. 2002. Biological activities of lavender essential oil. Phytother. Res. 16: 301-308.

23. Hsu CK, Chang CT, Lu HY, Chung YC. 2007. Inhibitory effects of the water extracts of Lavendula sp. on mushroom tyrosinase activity. Food Chem. 105: 1099-1105.

24. Kim AA, HA JH, Kim AR, Jeong HJ, Kim KM, Park SN. 2017. Cellular protective effect and active component analysis of lavender (Lavandula angustifolia) extracts and fractions. Appl. Chem. Eng. 28: 479-484.

25. Spiridon I, Colceru S, Anghel N, Teaca CA, Bodirlau R, Armatu A. 2011. Antioxidant capacity and total phenolic contents of oregano (Origanum vulgare), lavender (Lavandula angustifolia) and lemon balm (Melissa officinalis) from Romania. Nat. Prod. Res. 25: 1657-1661.

26. Ahn YJ, Won BR, Kang MK, Kim JH, Park SN. 2009. Antioxidant activity and component analysis of fermented Lavandula angustifolia extracts. J. Soc. Cosmet. Scientists Korea 35: 125-134.

27. Kim JK, Kim Y, Na KM, Surh YJ, Kim TY. 2007. [6]-Gingerol prevents UVB-induced ROS production and COX-2 expression in vitro and in vivo. Free Radic. Res. 41: 603-614.

28. Masaki H. 2010. Role of antioxidants in the skin: anti-aging effects. J. Dermatol. Sci. 58: 85-90.

29. Park K, Lee JH. 2008. Protective effects of resveratrol on UVB-irradiated $\mathrm{HaCaT}$ cells through attenuation of the caspase pathway. Oncol. Rep. 19: 413-417.

30. Im AR, Song JH, Lee MY, Yeon SH, Um KA, Chae S. 2014. Anti-wrinkle effects of fermented and non-fermented Cyclopia intermedia in hairless mice. BMC Complement. Altern. Med. 14: 424.

31. Gurung N, Ray S, Bose S, Rai V. 2013. A broader view: microbial enzymes and their relevance in industries, medicine, and beyond. Biomed. Res. Int. 2013: 329121.

32. Yu-Ling Wena, Li-Pyng Yan, Chen C-S. 2013. Effects of fermentation treatment on antioxidant and antimicrobial activities of four common Chinese herbal medicinal residues by Aspergillus oryzae. J. Food Drug Analysis. 21: 219-226.

33. Ortiz-Castro R, Contreras-Cornejo HA, Macias-Rodriguez L, Lopez-Bucio J. 2009. The role of microbial signals in plant growth and development. Plant Signal. Behav. 4: 701-712.

34. Antai SP, Obong US. 1992. The effect of fermentation on the nutrient status and on some toxic components of Icacinia manni. Plant Foods Hum. Nutr. 42: 219-224.

35. Sung BK, Chung JW, Bae HR, Choi JS, Kim CM, Kim ND, et al. 2105. Humulus japonicus extract exhibits antioxidative and anti-aging effects via modulation of the AMPK-SIRT1 pathway. Exp. Ther. Med. 9: 1819-1826.

36. Pervin M, Unno K, Nakamura Y, Imai S. 2016. Luteolin suppresses ultraviolet A- and B-induced matrix metalloproteinase 1- and 9 expression in human dermal fibroblast cells. J. Nutr. Food Sci. 6: 1-6.

37. Hwang YP, Oh KN, Yun HJ, Jeong HG. 2011. The flavonoids apigenin and luteolin suppress ultraviolet A-induced matrix metalloproteinase-1 expression via MAPKs and AP-1dependent signaling in HaCaT cells. J. Dermatol. Sci. 61: 23-31.

38. Apigenin induces dermal collagen synthesis via $\operatorname{smad} 2 / 3$ signaling pathway. Eur. J. Histochem. 59: 2467. 